\title{
INDICADORES DE INTERNACIONALIZAÇÃO: O QUE OS RANKINGS ACADÊMICOS MEDEM?
}

INDICATORS OF INTERNATIONALIZATION:

WHAT DO ACADEMIC RANKINGS MEASURE?

INDICADORES DE INTERNACIONALIZACIÓN

¿QUÉ MIDEN LOS RANKINGS ACADÉMICOS?

\section{Fernanda Geremias Leal Luciane Stallivieri \\ Mário César Barreto Moraes}

RESUMO: Um dos aspectos que reflete a importância da internacionalização da educação superior no contexto contemporâneo é criação de rankings acadêmicos globais e a inclusão de indicadores de internacionalização nessas ferramentas, que têm influenciado significativamente as políticas universitárias e governamentais. A despeito do prestigiado status adquirido pelos rankings, seus indicadores ainda refletem certa obscuridade e, nesse sentido, suscitam debates sobre sua capacidade de mensurar qualidade educacional. Diante desse cenário, o objetivo deste artigo é analisar os principais rankings acadêmicos internacionais e nacionais, com foco nos indicadores relacionados à internacionalização da educação superior. A pesquisa internacional, na forma de publicação, constitui-se como o indicador de internacionalização mais significativo para os rankings investigados, em parte devido a seu caráter quantitativo, que viabiliza a análise transnacional. Na ausência de análises qualitativas e contextualizadas, com indicadores e pesos adotados segundo justificações teóricas, os rankings acabam não contemplando a totalidade dos objetivos e missões que permeiam as universidades ao redor do mundo, e tampouco a complexidade inerente ao conceito de internacionalização da educação superior.

PALAVRAS-CHAVE: Internacionalização. Educação superior. Rankings acadêmicos.

ABSTRACT: One of the aspects that reflects the importance of the internationalization of higher education in the contemporary context is the creation of global academic rankings and the inclusion of internationalization indicators in these tools, which have significantly influenced university and government policies. In spite of the prestigious status acquired by the rankings, its indicators still reflect a certain obscurity and, in this sense, they provoke debates about its capacity to measure educational quality. Considering this scenario, the objective of this article is to analyze the main international and national academic rankings, focusing on indicators related to the internationalization of higher education. International research, in the form of publication, constitutes the most significant indicator of internationalization for the investigated rankings, partly due to its quantitative character, which makes the transnational analysis feasible. In the absence of qualitative and contextualized analyzes, with indicators and weights adopted according to theoretical justifications, the rankings end up not contemplating the totality of the objectives and missions that pervade the universities around the world, nor the complexity inherent to the concept of internationalization of higher education.

KEYWORDS: Internationalization. Higher education. Academic rankings.

RESUMEN: Uno de los aspectos que refleja la importancia de la internacionalización de la educación superior en el contexto contemporáneo es la creación de rankings académicos globales y la inclusión de indicadores de internacionalización en esas herramientas, que han influido significativamente en las políticas universitarias y

Submetido em: 19/08/2017 - Aceito em: 01/10/2017 - Publicado em: 09/10/2017.

\begin{tabular}{l|l|l|l|l|l|}
\hline (C) Rev. Inter. Educ. Sup. & Campinas, SP & v.4 & n.1 & p.52-73 & jan./abr. 2018 \\
\hline
\end{tabular}


gubernamentales. A pesar del prestigioso status adquirido por los rankings, sus indicadores todavía reflejan cierta oscuridad y, en ese sentido, suscitan debates sobre su capacidad de medir calidad educativa. Ante este escenario, el objetivo de este artículo es analizar los principales rankings académicos internacionales y nacionales, con foco en los indicadores relacionados a la internacionalización de la educación superior. La investigación internacional, en forma de publicación, se constituye como el indicador de internacionalización más significativo para los rankings investigados, en parte debido a su carácter cuantitativo, que viabiliza el análisis transnacional. La ausencia de análisis cualitativos y contextualizados, con indicadores y pesos adoptados según justificaciones teóricas, los rankings acaban no contemplando la totalidad de los objetivos y misiones que permean las universidades alrededor del mundo, y tampoco la complejidad inherente al concepto de internacionalización de la educación superior.

PALABRAS CLAVE: Internacionalización. Educación universitaria. Rankings académicos.

\section{INTRODUÇÃO}

O conceito de internacionalização adquiriu centralidade no campo da educação superior nos anos 1990 e, desde então, passou a integrar as políticas educacionais dos governos nacionais, assim como as estruturas e os planejamentos estratégicos das instituições universitárias de todo o mundo (LIMA; CONTEL, 2011; LAUS, 2012; DE WIT, 2016; STALLIVIERI, 2017).

Um dos aspectos que reflete a importância da internacionalização no contexto contemporâneo é criação de rankings acadêmicos globais e a inclusão de indicadores de internacionalização nesses rankings. Tratam-se de ferramentas voltadas à hierarquização das instituições universitárias, que, a partir de diferentes parâmetros avaliativos, visam a informar e a orientar estudantes, docentes e pesquisadores, a opinião pública e a sociedade em geral a respeito das melhores instituições universitárias presentes nas arenas nacional e internacional. Igualmente, podem servir como critério para a destinação de financiamento público e privado (PÉREZESPARRELLS; GARCIA, 2009) e, em uma perspectiva mais crítica, para acirrar a competitividade entre universidades e centros de pesquisa (NUNES; FERNANDES, 2014), reforçando o viés de produtivismo acadêmico e de instrumentalismo econômico que tem caracterizado a educação superior internacional contemporânea (VIEIRA; LIMA, 2016).

Os rankings acadêmicos têm influenciado de maneira significativa as políticas universitárias e governamentais (PÉREZ-ESPARRELLS; GÓMES-SANCHO, 2010; O’CONNELL; SAUNDERS, 2012; VIEIRA; LIMA, 2015). Nos ambientes em que as relações competitivas prevalecem, eles dão credibilidade às instituições ranqueadas e favorecem o financiamento público, o investimento privado e o aumento de matrículas de estudantes locais e internacionais nas universidades mais bem classificadas (VIEIRA; LIMA, 2015). Entretanto, a despeito do status adquirido, existe certa obscuridade em relação aos indicadores usados nessas avaliações, sobretudo no que diz respeito à sua capacidade para mensurar a qualidade educacional.

\begin{tabular}{l|l|l|l|l|l} 
(C) Rev. Inter. Educ. Sup. & Campinas, SP & v.4 & n.1 & p.52-73 & jan./abr. 2018 \\
\hline
\end{tabular}


Quando se trata de indicadores relacionados à internacionalização, essa falta de clareza se intensifica, em parte devido à complexidade inerente ao conceito e às dificuldades de compreendê-lo em profundidade, que resultam na falta de consenso sobre o seu significado e na inexistência de modelos padronizados para que as universidades ampliem sua dimensão internacional (KNIGHT, 2004, 2015; DE WIT, 2015). Como Knight (2015, p. 1, tradução nossa) observa, ainda "há muita confusão em relação a o que realmente significa uma universidade internacional, binacional, transnacional, cosmopolita, multinacional ou global".

Diante desse contexto, o objetivo deste artigo é analisar os principais rankings acadêmicos, com foco nos indicadores relacionados à internacionalização da educação superior. Seis ferramentas avaliativas ${ }^{2}$ são abrangidas pela análise, sendo três internacionais - Academic Rankings of World Universities (ARWU); Q\&S World University Rankings (Q\&S) e Times Higher Education World University Rankings (THE) - e três nacionais - Ranking Universitário Folha (RUF); Ranking Guia do Estudante (GE) e Conceito Preliminar de Curso (CPC) / Índice Geral de Cursos (IGC) -. Tais rankings adotam metodologias próprias e hierarquizam as universidades segundo seus critérios.

Trata-se de uma pesquisa de natureza qualitativa e descritiva, desenvolvido por meio de consultas bibliográficas e documentais. $O$ estudo visa a contribuir para a gestão das instituições universitárias brasileiras, no sentido de prestar orientações preliminares em relação aos rankings acadêmicos e subsidiar os planejamentos estratégicos e ações relativas à internacionalização. Em um primeiro momento, contextualiza-se a literatura relacionada aos rankings acadêmicos. Na sequência, aborda-se uma síntese das origens, das metodologias e dos eixos de avaliação dos rankings contemplados na análise, para, então, descrever-se como cada um deles avalia o quesito internacionalização. Por fim, apresentam-se algumas reflexões e as referências utilizadas.

\section{OS RANKINGS ACADÊMICOS}

Dois processos distintos devem ser considerados no debate sobre a avaliação da qualidade da educação superior. O primeiro, conduzido pelas agências de avaliação e acreditação, tem como principal objetivo subsidiar a tomada de decisão política dos respectivos governos. Trata-se de um processo complexo e multidimensional, que engloba aspectos como a

\footnotetext{
${ }^{2}$ Os rankings nacionais avaliam apenas as instituições universitárias do país de referência, enquanto os rankings internacionais, também chamados global rankings ou overall rankings, englobam as instituições universitárias de todos os países.
} 
qualidade do ensino, da pesquisa, da extensão e diversas outras categorias que compõem as estruturas universitárias (PÉREZ-ESPARRELLS; GARCÍA, 2009; VIEIRA; LIMA, 2015).

O segundo, objeto deste estudo, corresponde aos rankings acadêmicos, administrados por meios massivos de comunicação, governos e organizações sociais, com o objetivo de hierarquizar as instituições universitárias presentes nos cenários nacional e internacional. Trata-se de uma espécie de avaliação de reputação e prestígio, que não necessariamente reflete a qualidade das instituições avaliadas (PÉREZ-ESPARRELLS; GARCÍA, 2009; MARGINSON, 2016). Nunes e Fernandes (2014, p. 1), nesse aspecto, aponta que um dos sentidos dos rankings internacionais é "conferir legitimidade e notoriedade às instituições classificadas, a partir do que elas elegem como indicadores de excelência”. Marginson (2016, p. 1, tradução nossa) complementa que "os rankings fornecem um mapeamento claro de ‘quem é quem no zoológico' e, na melhor das hipóteses, servem como um guia objetivo para as principais concentrações da capacidade de pesquisa e de reputação, embora não informem onde as pessoas vão aprender mais ou melhor".

A origem dos rankings remonta ao final da década de 1950, nos Estados Unidos, sendo que o desenvolvimento das hierarquizações focadas na educação superior proliferou neste mesmo país início dos anos 1980, e posteriormente no Reino Unido, na década de 1990, com o propósito inicial de diferenciar as melhores Business Schools (PÉREZ-ESPARRELLS; GARCÍA, 2009; VIEIRA; LIMA, 2015). A publicação norte-americana World Report Best College, de 1983, foi uma das ferramentas criadas nesse contexto. A Unesco (2013) complementa que as publicações America's Best Colleges nos Estados Unidos, e Where we get our best men e Times Good University Guide, na Inglaterra, marcaram o início do interesse pelo tema.

Nos anos 2000 houve uma proliferação das ferramentas globais voltadas à classificação das universidades e centros de pesquisa. As instituições universitárias acompanharam esse movimento: diante das tendências contemporâneas de internacionalização e, impulsionadas pelo desejo de atrair os melhores cérebros para os seus quadros, passaram a direcionar seu olhar ao cumprimento dos quesitos contemplados pelos rankings e a buscar o status de “World Class University” (BUELA-CASAL et al., 2007; O'CONNELL; SAUNDERS, 2012; ALTBACH, 2015; DE WIT, 2016; VIEIRA; LIMA, 2016). Uma evidência desse fenômeno é a existência de consultorias e eventos voltados a essa finalidade, como o Brand U: How colleges and universities nurture their reputations, realizado pelo THE em setembro de 2016 (THE, 2016b).

Knight (2012, p. 30, tradução nossa), nesse aspecto, observa que apesar dos problemas destacados pela literatura no que diz respeito à supervalorização dos rankings, "reitores de universidades parecem usá-los para estruturar suas prioridades, declarando em seus planos

\begin{tabular}{l|c|c|c|c|c|}
\hline C Rev. Inter. Educ. Sup. & Campinas, SP & v.4 & n.1 & p.52-73 & jan./abr. 2018 \\
\hline
\end{tabular}


estratégicos que um resultado mensurável da internacionalização será a conquista de uma posição específica em um ou mais dos sistemas de classificação global". Maringe, Foskett e Woodfield (2013) confirmam essa tendência, ao constatarem que 90\% dos representantes de universidades anglófonas consideram a conquista de altas posições nos ranqueamentos internacionais como objetivo atrelado à internacionalização institucional. Altbach e Hazelkorn (2017) também seguem nessa linha, ao argumentarem que as universidades estão atualmente obcecadas em adquirir status nos rankings acadêmicos nacionais e internacionais, ainda que algumas delas jamais admitam isso.

Essa magnitude adquirida pelos rankings no campo da educação superior também pode ser evidenciada pela sua condição de objeto de análise por parte dos organismos internacionais envolvidos nesse contexto, sobretudo os europeus, como a European University Association (EUA); o Espaço Europeu do Ensino Superior (EEES); o Espaço Europeu de Investigação (EEI) e o Center for Higher Education (CHE), os quais têm produzido estudos e relatórios para compreender e apontar as possíveis consequências dessas ferramentas avaliativas (NUNES; FERNANDES, 2014). A Unesco também tem se preocupado com o tema e, em 2013, organizou uma publicação para discutir os desdobramentos dos rankings acadêmicos no contexto da educação superior (UNESCO, 2013). O tema tem suscitado, ainda, debates acadêmicos, como demonstram o tema e a programação do evento IREG-8 Conference: University rankings and international academic relations: a bridging tool or a hindrance?, realizado em maio de 2016 na Universidade Nova de Lisboa, em Lisboa, Portugal (IREG, 2016) e a publicação de artigos de periódicos sobre o tema.

Cada um dos rankings existentes adota uma metodologia própria, sem consenso em relação aos indicadores adotados (PÉREZ-ESPARRELLS; GÓMES-SANCHO, 2010), o que dificulta a avaliação comparativa direta. De acordo com Aguillo (2012), dois grandes grupos, que se diferenciam pela forma de obtenção dos dados, podem ser destacados: 1. Rankings baseados em pesquisas de opinião e 2. Rankings baseados em bibliometria ou cibermetria. Nunes e Fernandes (2014), por sua vez, evidencia que é comum que esses rankings gerem resultados bastante distintos entre si. Os rankings internacionais, especificamente, tendem a ser mais generalistas e a estarem voltados principalmente para a pesquisa. Uma vez que eles buscam incorporar o maior número possível de países - os quais distinguem-se significativamente em termos de características socioeconômicas e níveis de desenvolvimento - tais rankings concebem a produção científica, única passível de mensuração quantitativa em termos globais, como seu principal indicador (PÉREZ-ESPARELLS; GARCÍA, 2009; PÉREZ-ESPARRELLS; GÓMES-SANCHO, 2010).

Alguns autores argumentam que, quando elaborados a partir de critérios transparentes, os rankings podem servir como meio de prestações de contas para a sociedade (accountability); estimular o desenvolvimento de competências por parte das instituições universitárias;

\begin{tabular}{l|c|c|c|c|c|}
\hline C Rev. Inter. Educ. Sup. & Campinas, SP & v.4 & n.1 & p.52-73 & jan./abr. 2018 \\
\hline
\end{tabular}


subsidiar as decisões da gestão universitária em relação à cooperação internacional; complementar o trabalho de avaliação de qualidade feito pelas agências de avaliação e acreditação; bem como gerar um debate acerca dos elementos que efetivamente configuram a qualidade no campo da educação superior (PÉREZ-ESPARELLS; GARCÍA, 2009; O’CONNELL; SAUNDERS, 2012; UNESCO, 2013).

É pertinente reconhecer, contudo, as significativas contradições sociais que permeiam os processos de globalização e de internacionalização (LIMA; CONTEL, 2011) e a relação desse contexto mais amplo - no qual a competitividade ocorre em condições de desigualdade - com os rankings acadêmicos (MARGINSON, 2017). Deve-se considerar, ainda, as limitações inerentes às metodologias adotadas por tais ferramentas, que, controversas e subjetivas (LIU, 215), estão intrinsecamente conectadas à comercialização da educação superior, e, portanto, podem induzir a concepções errôneas acerca das instituições avaliadas (PÉREZESPARELLS; GARCÍA, 2009), reforçando o capitalismo acadêmico (VIEIRA; LIMA, 2016; KIM, 2017) e as geografias desiguais de educação superior (JONS; HOYLER, 2013).

\section{RANKINGS ACADÊMICOS INTERNACIONAIS}

\section{Academic Rankings of World Universities (ARWU)}

O Academic Rankings of World Universities (ARWU), também conhecido como o Ranking de Shanghai, foi criado pela Shanghai Jiao Tong University em 2003, como parte do esforço chinês de alcançar a excelência em suas universidades. O Projeto 985, resultante da declaração do presidente da China em 1998 de que o país deveria ter universidades de nível internacional (world-class universities) - em parte como forma de diminuir o fenômeno de fuga dos cérebros (brain drain) -, investiu inicialmente em nove instituições universitárias, entre elas a Shanghai Jiao Tong University, a qual, a partir de um estudo comparativo das universidades chinesas com as universidades norte-americanas mais importantes, estabeleceu critérios de excelência para ranquear as universidades do mundo (LIU, 2015).

O ARWU publica anualmente a lista das 500 melhores universidades e avalia e classifica cerca de 1200 instituições (ARWU, 2016). Os cinco campos do conhecimento contemplados são: Ciências Naturais e Matemática; Engenharia/Tecnologia e Ciências da Computação; Ciências da Vida e Agricultura; Medicina Clínica e Farmácia; e Ciências Sociais. Segundo Liu (2015), os campos de Artes e Humanidades, de Psicologia e de outras ciências interdisciplinares não fazem parte do ARWU devido às dificuldades de se estabelecer indicadores internacionalmente comparáveis (LIU, 2015; NUNES; FERNANDES, 2014). 
Seis indicadores objetivos são utilizados pelo ARWU na classificação das universidades do mundo: 1. Número de ex-alunos e de docentes/pesquisadores vencedores de prêmios Nobel e de medalhas Fields (peso de 10\% para ex-alunos e de $20 \%$ para docentes/pesquisadores); 2. Número de pesquisadores altamente citados pela Thomson Reuters (peso de 20\%); 3. Número de artigos publicados nos periódicos da Nature e da Science na última década (peso de 20\%); 4. Número de artigos indexados ao Science Citation Index (SCIE); 5. Número de artigos indexados ao Expanded - Social Science Citation Index (SSCI) (peso de 20\%) e 6. Desempenho docente per capita da universidade (peso de 10\%) (ARWU, 2016).

Evidencia-se, portanto, que, de maneira geral, o ARWU classifica as universidades principalmente a partir do critério de desempenho na pesquisa. Embora ele tenha sido considerado coerente e transparente pela revista The Economist em 2005 (NUNES; FERNANDES, 2014; ARWU, 2016), alguns problemas de natureza metodológica e técnica podem ser evidenciados. Entre eles, a falta de equilíbrio entre a pesquisa e o ensino; a exclusão de indicadores como publicações em outros idiomas que não o Inglês, a inclinação às ciências (LIU, 2015; NUNES; FERNANDES, 2014). Por tratar-se de uma avaliação fortemente voltada à pesquisa, que desconsidera a formação, este ranking tem sido muito criticado (JONS; HOYLER, 2013). Em 2010, os ministros de educação da França, da Noruega e da Dinamarca visitaram a Shanghai Jiao Tong University para discutir maneiras de aprimorar a metodologia adotada (NUNES; FERNANDES, 2014).

De acordo com Liu (2015), o ARWU tem levantado possibilidades de prover listas de ranqueamento mais diversificadas, que considerem os diferentes tipos de universidades existentes e incluam elementos como características disciplinares, história, tamanho da instituição e orçamento disponível. Além da hierarquização geral das universidades do mundo, o ARWU passou a promover outros dois ranqueamentos mais específicos: $A R W U$ Field (por campo do conhecimento) e ARWU Subject (por disciplina) (ARWU, 2016).

\section{Q\&S World University Rankings (Q\&S)}

O Q\&S World University Rankings é um ranking publicado desde 2004 pela Quacquarelli Symonds, empresa britânica especializada em educação internacional. Durante o período de 2004 a 2009, o Q\&S foi publicado pela Times Higher Education, revista que trata de questões relacionadas à educação superior. Em 2010, o Q\&S e a Times Higher Education finalizaram o trabalho conjunto, de modo que o Q\&S assumiu uma publicação com a metodologia já adotada, enquanto a Times Higher Education criou uma nova metodologia, que deu origem à publicação Times Higher Education World University Rankings (THE).

O Q\&S contempla os campos disciplinares Ciências Naturais; Biomedicina; Tecnologia; Ciências Sociais e Artes e Humanidades. Seis indicadores são considerados por este ranking

\begin{tabular}{l|l|l|l|l|l} 
(C) Rev. Inter. Educ. Sup. & Campinas, SP & v.4 & n.1 & p.52-73 & jan./abr. 2018 \\
\hline
\end{tabular}


na classificação das melhores universidades: 1. Reputação acadêmica segundo um peer review global (peso de 40\%); 2. Reputação segundo empregadores globais (peso de 10\%); 3. Proporção de estudantes por faculdade (peso de 20\%); 4. Citações por faculdade na base Scopus (peso de 20\%); 5. Proporção de estudantes internacionais (peso de 5\%) e 6. Proporção de docentes internacionais (peso de 5\%). Ao todo, mais de 4000 instituições são consideradas e mais de 900 são ranqueadas (Q\&S, 2016).

Verifica-se, portanto, que o quesito reputação é o mais significativo para o Q\&S, o qual é determinado por meio de entrevistas com docentes e pesquisadores (peer review), que indicam até trinta instituições universitárias que eles consideram mais importantes nos campos contemplados, bem como por meio de entrevistas com gestores de recursos humanos de empresas globais, que informam de quais universidades os egressos são mais frequentemente recrutados (NUNES; FERNANDES, 2014; Q\&S, 2016). Além do $Q \& S$ World University Rankings, referente à hierarquização geral das universidades do mundo, a Q\&S passou a promover outros ranqueamentos mais específicos: Q\&S World University Rankings by subject (por disciplina); Q\&S Graduate Employability Rankings (por empregabilidade); $Q \& S$ Best Students Cities (por melhores cidades para estudantes) além dos ranqueamentos regionais (região árabe; Ásia; BRICS; Europa emergente e Ásia Central; América Latina) (Q\&S, 2016).

\section{Times Higher Education World University Rankings (THE)}

O Times Higher Education World University Rankings (THE), fundado em 2004 e publicado separadamente do Q\&S desde 2010, é atualmente desenvolvido em parceria com a Elsevier. Diferentemente dos ARWU, que utiliza somente dados secundários, o THE solicita informações às próprias instituições universitárias. Os seguintes campos disciplinares são contemplados por esta avaliação: Artes e Humanidades; Ciências Sociais; Ciências Físicas; Ciências da Vida; Engenharia e Tecnologia; além de Medicina. Ao todo, mais de 900 universidades de cerca de 80 países são ranqueadas (THE, 2016).

Treze indicadores de desempenho são atualmente considerados pelo THE, os quais são agrupados em cinco grandes áreas: 1 . Ensino (ambiente de aprendizagem), com peso de 30\%, sendo $15 \%$ referente à pesquisa de reputação; 4,5\% referente à proporção de funcionário por estudante, $2,25 \%$ referente à proporção de doutorando por bacharel, $6 \%$ referente à proporção de títulos de doutor concedidos por docente e 2,25\% referente ao orçamento da instituição; 2. Pesquisa (volume, orçamento e reputação), com peso de $30 \%$, sendo $18 \%$ referente à pesquisa de reputação; $6 \%$ referente ao orçamento para pesquisa e $6 \%$ referente à produtividade de pesquisa; 3. Citações (influência da pesquisa), com peso de 30\%; 4. Perspectiva internacional (docentes, estudantes e pesquisadores estrangeiros e colaboração internacional), com peso de $7,5 \%$, sendo $2,5 \%$ referente à proporção de estudantes estrangeiros por estudantes

\begin{tabular}{l|l|l|l|l|l}
\hline (c) Rev. Inter. Educ. Sup. & Campinas, SP & v.4 & n.1 & p.52-73 & jan./abr. 2018 \\
\hline
\end{tabular}


domésticos, 2,5\% referente à proporção de estudantes estrangeiros por funcionários domésticos e 2,5\% referente à colaboração internacional e 5. Orçamento da indústria (transferência de conhecimento), com peso de 2,5\% (THE, 2016a).

Assim como o ARWU e o Q\&S, o THE também passou a publicar, além da hierarquização geral das universidades do mundo, outros ranqueamentos mais específicos: THE BRICS \& Emerging Economies Rankings (por países dos BRICS e economias emergentes); THE World Reputation Rankings (por reputação) e THE Asia University Rankings (Ásia), THE Latin America Rankings (América Latina) (THE, 2016).

\section{RANKINGS ACADÊMICOS NACIONAIS}

\section{Ranking Universitário Folha (RUF)}

O Ranking Universitário Folha (RUF) é uma avaliação do ensino superior brasileiro desenvolvido pela Folha desde 2012, que engloba tanto a hierarquização das universidades quanto a hierarquização dos cursos. Os dados são coletados por uma equipe da Folha em bases de patentes brasileiras, de periódicos científicos, do Ministério da Educação (MEC) e em pesquisas nacionais de opinião. 192 universidades brasileiras, públicas e privadas, bem como todos os cursos de graduação existentes no Brasil, fazem parte da avaliação (RUF, 2015, 2015a). Também são ranqueadas 2391 Instituições de Ensino Superior (IES), considerando como critério básico a procura de estudantes pelos cursos. De acordo com o Datafolha (RUF, 2015), a inclusão de dados a respeito de egressos e da extensão universitária encontra-se em estudo.

Os indicadores contemplados pelo RUF na hierarquização das universidades são: 1. Pesquisa (máximo de 42 pontos, sendo 7 referentes ao número de trabalhos científicos publicados, 7 referentes ao número de citações, 7 referentes à proporção de publicações por docente, 7 referentes à proporção de citações por docente, 5 referentes à proporção de citações por publicação, 4 referentes ao volume de recursos obtidos por agências de fomento, 3 referentes ao número de publicações em periódicos nacionais e 2 referentes à proporção de pesquisadores com alta produção acadêmica); 2. Internacionalização (total de 4 pontos, sendo 2 referentes ao número de citações de trabalhos da instituição por grupos internacionais e 2 referentes à proporção de publicações da universidade em coautoria internacional); 3 . Inovação (total de 4 pontos, referentes ao número de pedidos de patentes); 4 . Ensino (total de 32 pontos, sendo 22 referentes a pesquisa feita pelo Datafolha com 726 professores que analisam a qualidade dos cursos superiores, 4 referentes à proporção de professores com doutorado e com mestrado, 4 referentes à proporção de professores em regime de dedicação 
exclusiva e 2 referentes ao desempenho do aluno no Enade) e 5. Mercado (total de 18 pontos, referentes a pesquisa feita pelo Datafolha com responsáveis pela contratação de profissionais no mercado) (RUF, 2015a).

\section{Ranking Guia do Estudante (GE)}

Existente desde 1984, o Guia do Estudante (GE) é uma pesquisa de opinião promovida pela Editora Abril e destinada a orientar as pessoas interessadas em ingressar em cursos universitários sobre o que e onde estudar; apresentar informações atualizadas sobre o mercado de trabalho e novas profissões; fornecer orientações sobre pós-graduação e bolsas de estudos e reunir uma lista com o nome e o endereço das faculdades e universidades do Brasil (GUIA DO ESTUDANTE, 2016).

A partir de 1988, o GE passou a classificar os cursos superiores brasileiros por meio de um sistema de estrelas, variável de 1 (curso fraco) a 5 (curso excelente). Para serem avaliados, os cursos devem atender a quatro critérios: 1. Contemplar a titulação de bacharelado e licenciatura (com exceção de Pedagogia e Educação Física); 2. Ter uma turma formada pelo menos desde 2013; 3 . Ter turmas em andamento e ser oferecido no próximo processo seletivo e 4. Ser um curso presencial (GUIA DO ESTUDANTE, 2016, 2016a).

O processo de avaliação leva nove meses e constitui-se de cinco etapas: 1. Atualização dos dados das instituições: a redação do GE entra em contato com as mais de 2000 IES do país, que informam os cursos que serão ofertados no próximo vestibular; 2. Definição dos cursos que serão avaliados com base nos critérios estabelecidos; 3. Preenchimento de um formulário por parte dos coordenadores dos cursos, composto por quinze questões de temas relativos a: a) corpo docente; b) projeto pedagógico; c) produção científica; d) infraestrutura; e) inserção dos egressos no mercado de trabalho; f) internacionalização e g) oferta de pós-graduação (GUIA DO ESTUDANTE, 2016a).

Esse formulário não é pontuado para fins de avaliação, mas constitui-se como instrumento importante à medida que norteia os pareceristas no processo de avaliação; 4. Pesquisa de opinião com os pareceristas: equipe de mais de 6.500 coordenadores de cursos, diretores de departamentos e professores, que classificam os cursos conforme os conceitos: prefiro não opinar; ruim (1 estrela); regular (2 estrelas); bom (3 estrelas); muito bom (4 estrelas) e excelente (5 estrelas). Cada parecerista avalia cerca de 20 cursos distribuídos aleatoriamente, sendo que eles não podem avaliar os cursos das instituições onde trabalham; 5. Atribuição dos conceitos: a partir dos conceitos dados pelos consultores, o GE, com apoio do Ibope Inteligência (que presta consultoria técnica na coleta, sistematização e análise de dados) e da PricewaterhouseCoopers (que verifica todo o resultado da pesquisa), faz uma média dos conceitos que cada curso recebeu. Desde 2007, também são consideradas as avaliações dos

\begin{tabular}{|l|c|c|c|c|c|}
\hline CC Rev. Inter. Educ. Sup. & Campinas, SP & v.4 & n.1 & p.52-73 & jan./abr. 2018 \\
\hline
\end{tabular}


cinco anos anteriores. Como resultado, cada curso avaliado recebe um conceito, sendo divulgados aqueles com 3, 4 ou 5 estrelas (GUIA DO ESTUDANTE, 2016, 2016b).

Com base na avaliação dos consultores para os melhores cursos, em 2002 O GE passou a ranquear as melhores universidades e, em 2005, criou o Prêmio Melhores Universidades, de periodicidade anual, do qual as instituições participantes com pelo menos dois cursos estrelados podem participar. O ranqueamento das melhores universidades é dividido nas categorias "universidade do ano: escola pública e privada" e "as melhores por área do conhecimento", que contempla uma instituição pública e uma instituição privada em oito áreas (GUIA DO ESTUDANTE, 2016a, 2016b). Cabe enfatizar que a avaliação dos pareceristas é feita com base nos seguintes indicadores: 1. Questionário preenchido pelos coordenadores e 2. Notório saber dos pareceristas (conhecimento prévio sobre a instituição e o curso avaliados). Por tratar-se de pesquisa de opinião, os resultados refletem, principalmente, a imagem do curso perante a comunidade acadêmica (GUIA DO ESTUDANTE, 2016b).

\section{Rankings oriundos do CPC e do IGC}

Estes rankings são elaborados pelo Instituto Nacional de Estudos e Pesquisas Educacionais Anísio Teixeira (INEP) do Ministério da Educação (MEC) a partir do Conceito Preliminar de Curso (CPC), do Índice Geral de Cursos (IGC) e da divulgação dos resultados do Exame Nacional de Desempenho do Estudante (ENADE), uma prova obrigatória de conhecimentos gerais e específicos existente desde 2004 e aplicada com periodicidade máxima de três anos, que integra o Sistema Nacional de Avaliação da Educação Superior (SINAES).

O ENADE avalia o rendimento dos concluintes dos cursos de graduação de Instituições de Ensino Superior brasileiras em relação aos conteúdos programáticos, às habilidades e às competências adquiridas em sua formação, constituindo-se como um indicador de qualidade correspondente a $20 \%$ da avaliação dos cursos (MORAES, 2016). De acordo com Moraes (2016, p. 148), o CPC e o IGC são indicadores que "se destinam a mensurar a qualidade dos cursos e das instituições do país, sendo utilizados tanto para o desenvolvimento de políticas públicas da educação superior quanto como fonte de consultas pela sociedade”.

O Questionário do Estudante preenchido pelos participantes do ENADE e os dados do Censo da Educação Superior preenchidos pelas respectivas IES geram os insumos que servem como base para o cálculo do CPC, que se propõe a avaliar os cursos superiores brasileiros. Os indicadores contemplados pelo CPC, conforme orientação técnica aprovada pela Comissão Nacional de Avaliação da Educação Superior (CONAES), são: 1. Avaliação de desempenho de estudantes, com peso de 55\%, sendo $20 \%$ referente à nota dos concluintes e $35 \%$ referente ao Indicador de Diferença dentre os Desempenhos Observado e Esperado (IDD); 2. Corpo

\begin{tabular}{l|l|l|l|l|l|} 
(C) Rev. Inter. Educ. Sup. & Campinas, SP & v.4 & n.1 & p.52-73 & jan./abr. 2018 \\
\hline
\end{tabular}


docente, com peso de $30 \%$, sendo $15 \%$ referente à titulação de doutor, $7,5 \%$ referente à titulação de mestre e 7,5\% referente ao regime de trabalho; 3. Infraestrutura, com peso de 7,5\%; 4. Recursos didático-pedagógicos, com peso de 5\% e 5. Oportunidades de ampliação da formação acadêmica e profissional, com peso de 2,5\% (MORAES, 2016).

O IGC, por sua vez, se refere a "uma média ponderada dos conceitos dos cursos de graduação e pós-graduação stricto sensu da instituição" (MORAES, 2016, p. 163), que serve como referencial orientador das comissões de avaliação institucional. Para isso, utiliza: 1. A média dos últimos CPCs disponíveis dos cursos avaliados da instituição no ano do cálculo e nos dois anteriores; 2. A média dos conceitos de avaliação da CAPES dos programas de pósgraduação stricto sensu e 3. A distribuição dos alunos da IES entre seus diferentes níveis de ensino (graduação, mestrado e doutorado). Uma vez ajustados, os conceitos da graduação e da pós-graduação são ponderados de acordo com o número de alunos por curso em relação ao número total de alunos da IES (MORAES, 2016).

\section{SÍNTESE DAS PARTICULARIDADES DOS RANKINGS ACADÊMICOS}

Evidencia-se que cada um dos rankings analisados considera diferentes campos disciplinares e indicadores, com pesos distintos, na sua avaliação. Eles também se diferem em termos de estrutura e de procedimentos metodológicos adotados. As principais características dessas ferramentas são demonstradas, em perspectiva comparativa, no Quadro 1:

Quadro 1. Principais características dos rankings acadêmicos selecionados para o estudo

\begin{tabular}{|c|c|c|c|}
\hline \multicolumn{4}{|c|}{ Internacionais } \\
\hline Ranking & ARWU & $\mathbf{Q} \& \mathbf{S}$ & THE \\
\hline Ano de Criação & 2003 & 2004 & 2010 \\
\hline País de Origem & China & Reino Unido & Reino Unido \\
\hline $\begin{array}{l}\text { Instituição e } \\
\text { tipo de } \\
\text { instituição }\end{array}$ & $\begin{array}{l}\text { Shangai Jiao Tong University } \\
\text { (Universidade) }\end{array}$ & $\begin{array}{l}\text { Quacquerelli Simonds } \\
\text { (empresa privada } \\
\text { especializada em educação } \\
\text { internacional) }\end{array}$ & $\begin{array}{l}\text { Times Higher Education } \\
\text { (revista especializada em } \\
\text { educação internacional) e } \\
\text { Elsevier }\end{array}$ \\
\hline Periodicidade & Anual & Anual & Anual \\
\hline $\begin{array}{l}\text { Quantidade de } \\
\text { instituições } \\
\text { ranqueadas } \\
\end{array}$ & $\begin{array}{l}2000 \text { consideradas, sendo } 1200 \\
\text { classificadas e } 500 \text { publicadas. }\end{array}$ & $\begin{array}{l}\text { Mais de } 4000 \text { consideradas, } \\
\text { sendo mais de } 900 \\
\text { ranqueadas. }\end{array}$ & $\begin{array}{l}\text { Mais de } 900 \text { de cerca de } \\
80 \text { países ranqueadas. }\end{array}$ \\
\hline $\begin{array}{c}\text { Campos } \\
\text { disciplinares } \\
\text { contemplados }\end{array}$ & $\begin{array}{l}\text { - Ciências Naturais e } \\
\text { Matemática; } \\
\text { - Engenharia/Tecnologia e } \\
\text { Ciências da Computação; } \\
\text { - Ciências da Vida e Agricultura; } \\
\text { - Medicina Clínica e Farmácia; } \\
\text { - Ciências Sociais. }\end{array}$ & $\begin{array}{l}\text { - Ciências Naturais; } \\
\text { - Biomedicina; } \\
\text { - Tecnologia; } \\
\text { - Ciências Sociais; } \\
\text { - Artes e Humanidades. }\end{array}$ & $\begin{array}{l}\text { - Artes e Humanidades; } \\
\text { - Ciências Sociais; } \\
\text { - Ciências Físicas; } \\
\text { - Ciências da Vida; } \\
\text { - Engenharia e } \\
\text { Tecnologia; } \\
\text { - Medicina. }\end{array}$ \\
\hline Indicadores $e$ & - Número de ex-alunos (10\%) e & - Reputação acadêmica & - Ensino (ambiente de \\
\hline (C) Rev. Inter. & Campinas, SP & p.52-73 & jan./abr. 2018 \\
\hline
\end{tabular}




\begin{tabular}{|c|c|c|c|}
\hline $\begin{array}{l}\text { respectivos } \\
\text { pesos }(\%)\end{array}$ & $\begin{array}{l}\text { de docentes / pesquisadores } \\
\text { (20\%) vencedores de prêmios } \\
\text { Nobel e de medalhas Fields; } \\
\text { - Número de pesquisadores } \\
\text { frequentemente citados pela } \\
\text { Thomson Reuters (20\%); } \\
\text { - Número de artigos publicados } \\
\text { nos periódicos Nature e Science } \\
\text { (20\%); } \\
\text { - Número de artigos indexados ao } \\
\text { Science Citation Index; } \\
\text { - Número de artigos indexados ao } \\
\text { Expanded - Social Science } \\
\text { Citation Index (20\% para } \\
\text { ambos); } \\
\text { - Desempenho per capita da } \\
\text { universidade (10\%). }\end{array}$ & $\begin{array}{l}\text { segundo um peer review } \\
\text { global }(40 \%) ; \\
\text { - Reputação segundo } \\
\text { empregadores globais } \\
(10 \%) ; \\
\text { - Proporção de estudantes } \\
\text { por faculdade (20\%); } \\
\text { - Citações por faculdade na } \\
\text { base Scopus (20\%); } \\
\text { - Proporção de estudantes } \\
\text { internacionais }(5 \%) ; \\
\text { - Proporção de docentes } \\
\text { internacionais }(5 \%) \text {. }\end{array}$ & $\begin{array}{l}\text { aprendizagem) }(30 \%) \text {; } \\
\text { - Pesquisa (volume, } \\
\text { orçamento e reputação) } \\
(30 \%) \\
\text { - Citações (influência da } \\
\text { pesquisa) }(30 \%) ; \\
\text { - Perspectiva } \\
\text { internacional } \\
\text { (docentes, estudantes e } \\
\text { pesquisadores } \\
\text { estrangeiros e } \\
\text { colaboração } \\
\text { internacional) }(7,5 \%) ; \\
\text { - Orçamento da indústria } \\
\text { (2,5\%). }\end{array}$ \\
\hline $\begin{array}{c}\text { Principal } \\
\text { critério }\end{array}$ & Pesquisa & Reputação & Pesquisa $^{3}$ \\
\hline \multicolumn{4}{|c|}{ Nacionais } \\
\hline Ranking & RUF & GE & CPC/IGC \\
\hline Ano de Criação & 2012 & 1988 & 2007 \\
\hline País de Origem & Brasil & Brasil & Brasil \\
\hline $\begin{array}{c}\text { Instituição e } \\
\text { tipo de } \\
\text { instituição }\end{array}$ & $\begin{array}{l}\text { Folha (empresa privada de } \\
\text { comunicação) }\end{array}$ & $\begin{array}{l}\text { Abril (empresa privada de } \\
\text { comunicação) }\end{array}$ & $\begin{array}{l}\text { INEP/MEC (Governo } \\
\text { Brasileiro) }\end{array}$ \\
\hline Periodicidade & Anual & Anual & Trienal $^{4}$ \\
\hline $\begin{array}{l}\text { Quantidade de } \\
\text { instituições } \\
\text { ranqueadas } \\
\end{array}$ & Mais de 190 ranqueadas. & $\begin{array}{l}\text { Todos os cursos nacionais } \\
\text { que atenderem aos quatro } \\
\text { quesitos do GE. }\end{array}$ & Todas as IES nacionais. \\
\hline $\begin{array}{c}\text { Campos } \\
\text { disciplinares } \\
\text { contemplados } \\
\end{array}$ & $\begin{array}{l}\text { - Todos os cursos de graduação } \\
\text { existentes no Brasil. }\end{array}$ & $\begin{array}{l}\text { - Todos os cursos de } \\
\text { graduação existentes no } \\
\text { Brasil. }\end{array}$ & $\begin{array}{l}\text { - Todos os cursos de } \\
\text { graduação existentes } \\
\text { no Brasil. }\end{array}$ \\
\hline $\begin{array}{l}\text { Indicadores e } \\
\text { respectivos } \\
\text { pesos }(\%)\end{array}$ & $\begin{array}{l}\text { - Pesquisa }(42 \%) \text {; } \\
\text { - Internacionalização (4\%); } \\
\text { - Inovação }(4 \%) \text {; } \\
\text { - Ensino }(32 \%) \\
\text { - Mercado }(18 \%) \text {. }\end{array}$ & $\begin{array}{l}\text { - Corpo docente; } \\
\text { - Projeto pedagógico; } \\
\text { - Produção científica; } \\
\text { - Infraestrutura; } \\
\text { - Inserção dos alunos no } \\
\text { mercado de trabalho; } \\
\text { - Internacionalização; } \\
\text { - Oferta de pós- } \\
\text { graduação. }\end{array}$ & $\begin{array}{l}\text { CPC: } \\
\text { - Avaliação de } \\
\text { desempenho dos } \\
\text { estudantes }(55 \%) ; \\
\text { - Corpo docente }(30 \%) ; \\
\text { - Infraestrutura }(7,5 \%) ; \\
\text { - Recursos didático- } \\
\text { pedagógicos }(5 \%) ; \\
\text { - Oportunidades de } \\
\text { ampliação da formação } \\
\text { acadêmica e } \\
\text { profissional (2,5\%). } \\
\text { IGC: } \\
\text { - Média dos últimos } \\
\text { CPCs disponíveis dos }\end{array}$ \\
\hline
\end{tabular}

${ }^{3}$ Para fins de análise, no caso do THE consideramos o indicador citações como pesquisa, de modo que o percentual de $30 \%$ para citações e $30 \%$ para pesquisa totalizou $60 \%$.

${ }^{4} \mathrm{O}$ IGC é realizado anualmente, mas divulgado a cada três anos, juntamente com o CPC.

${ }^{5}$ O GE não explicita o peso atribuído para cada indicador considerado em sua avaliação.

\begin{tabular}{l|c|c|c|c|}
\hline (C) Rev. Inter. Educ. Sup. & Campinas, SP & v.4 & n.1 & p.52-73 \\
\hline
\end{tabular}
jan./abr. 2018 


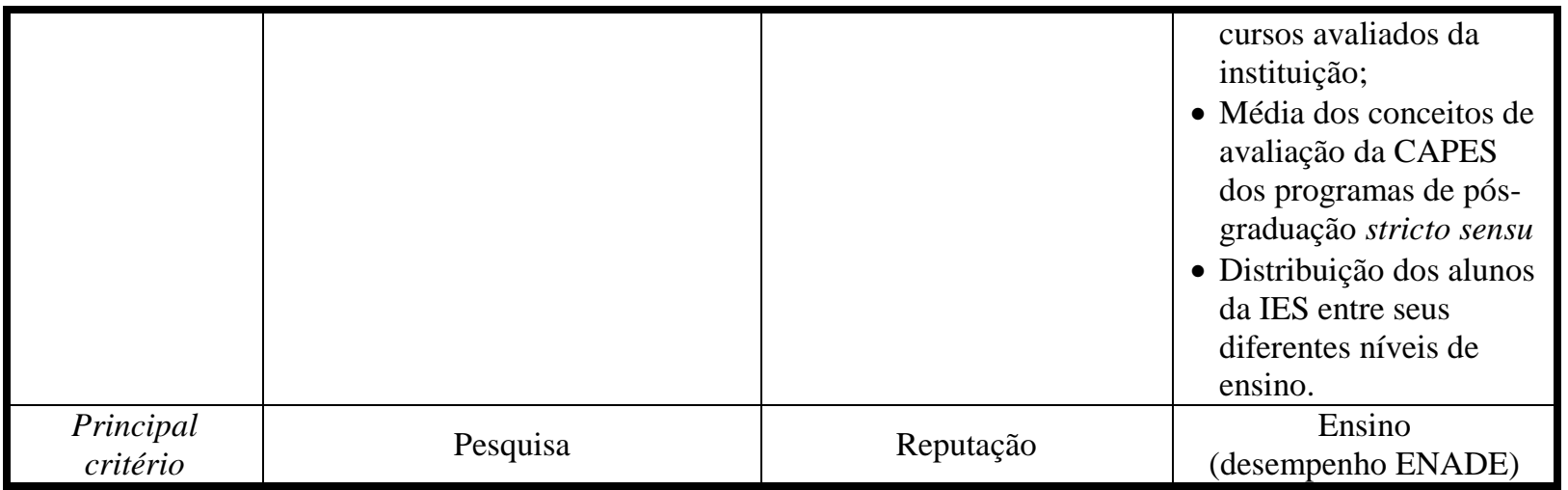

Fonte: Dados da pesquisa (2017).

Como o Quadro 1 demonstra, apesar das inúmeras diferenças entre os rankings acadêmicos, a maior parte deles considera a pesquisa como principal critério de avaliação. Dos rankings internacionais, apenas o Q\&S está mais preocupado com a reputação acadêmica, sendo, portanto mais subjetivo, ainda que também considere elementos relacionados à pesquisa. No contexto nacional, cada um dos três rankings abrangidos no recorte da pesquisa apresenta um diferente principal critério de análise: enquanto o RUF baseia-se principalmente na pesquisa, o GE reflete a imagem dos cursos e das instituições segundo a comunidade acadêmica e o CPC/IQS concentra-se sobretudo no desempenho dos estudantes no ENADE. Comparativamente, o THE e o RUF são os rankings que contemplam em termos mais proporcionais as funções universitárias de ensino e pesquisa, sendo que nenhum dos seis rankings, nacionais ou internacionais, parecem considerar atividades relativas à extensão em suas análises.

\section{INDICADORES RELACIONADOS À INTERNACIONALIZAÇÃO DA EDUCAÇÃO SUPERIOR}

Cinco dos seis rankings acadêmicos considerados no recorte da pesquisa utilizam como critérios de análise aspectos relacionados à internacionalização, ainda que alguns o façam de maneira indireta (ARWU) e outros o façam diretamente (Q\&S; THE; RUF; GE). O ranking nacional GE, apesar de considerar a internacionalização como um critério, não especifica, nas bases consultadas, quais indicadores são utilizados e tampouco o peso atribuído a essa dimensão.

O Quadro 2 apresenta os indicadores e os pesos que o Q\&S, o THE e o RUF explicitamente relacionam à internacionalização:

Quadro 2. Critérios de avaliação dos rankings acadêmicos relacionados à internacionalização

n.1

p.52-73




\begin{tabular}{|c|c|c|c|}
\hline Ranking & Q\&S & THE & RUF \\
\hline $\begin{array}{c}\text { Indicadores de } \\
\text { internacionalização e } \\
\text { respectivo peso }(\%)\end{array}$ & $\begin{array}{l}\text { - Proporção de } \\
\text { estudantes } \\
\text { internacionais (5\%); } \\
\text { - Proporção de } \\
\text { docentes } \\
\text { internacionais }(5 \%)\end{array}$ & $\begin{array}{l}\text { - Proporção de estudantes } \\
\text { estrangeiros por estudantes } \\
\text { domésticos }(2,5 \%) ; \\
\text { - Proporção de estudantes } \\
\text { estrangeiros por } \\
\text { funcionários domésticos } \\
(2,5 \%) ; \\
\text { - Colaboração internacional } \\
(2,5 \%)\end{array}$ & $\begin{array}{l}\text { - Número de citações de } \\
\text { trabalhos da instituição } \\
\text { por grupos internacionais } \\
(2 \%) ; \\
\text { - Proporção de publicações } \\
\text { da universidade em } \\
\text { coautoria internacional } \\
(2 \%) \text {. }\end{array}$ \\
\hline $\begin{array}{l}\text { Percentual destinado à } \\
\text { internacionalização }\end{array}$ & $10 \%$ & $7,5 \%$ & $4 \%$ \\
\hline
\end{tabular}

Fonte: Dados da pesquisa (2017).

O Quadro 2 demonstra que, comparativamente, o Q\&S é o ranking com maior percentual de indicadores explicitamente relacionados à internacionalização (10\%). No entanto, tais indicadores se limitam à presença internacional. Cabe mencionar que esta ferramenta, de certa forma, também considera a pesquisa internacional em sua análise, por meio do indicador "citação por faculdade na base Scopus", com peso de 20\%.

O THE, por sua vez, apesar de um apresentar percentual menor de indicadores explicitamente relacionados à internacionalização $(7,5 \%)$, considera tanto elementos de presença internacional quanto de colaboração internacional para a pesquisa. Seus dois primeiros indicadores, "proporção de estudantes estrangeiros por estudantes domésticos" e "proporção de estudantes estrangeiros por funcionários domésticos", refletem a visão do THE de que a habilidade da universidade de atrair estudantes de graduação, pós-graduação e docentes de todo o mundo é chave para o seu sucesso em nível mundial (THE, 2016a). O terceiro indicador, "colaboração internacional", é oriundo do cálculo proporcional do total de publicações em periódicos que tenham pelo menos um coautor estrangeiro (THE, 2016a).

O RUF apresenta como indicadores de internacionalização apenas elementos relacionados à pesquisa. O ARWU, por sua vez, não contempla indicadores explicitamente relacionados à internacionalização, no entanto, seus indicadores estão fortemente centrados na pesquisa internacional, equivalendo-se, assim, aos indicadores que os demais rankings explicitamente consideram como indicadores de internacionalização.

Evidencia-se, desse modo, que de maneira geral os indicadores de internacionalização presentes nos rankings acadêmicos são relativos à mobilidade internacional, às parcerias internacionais para a pesquisa e às citações em artigos científicos internacionais, os quais são mais facilmente quantificáveis e comparáveis. Contudo, cabe lembrar que a internacionalização da educação superior é um fenômeno abrangente, com diferentes significados para diferentes pessoas, que é impulsionado por uma diversidade de motivações

\begin{tabular}{|l|c|c|c|c|c|}
\hline (C) Rev. Inter. Educ. Sup. & Campinas, SP & v.4 & n.1 & p.52-73 & jan./abr. 2018 \\
\hline
\end{tabular}


e que se manifesta diferentemente nos diversos contextos onde ocorre (KNIGHT, 2004, 2015; MARGINSON, 2016).

Em termos de indicadores, além daqueles contemplados pelos rankings, a internacionalização envolve a colaboração internacional para outras finalidades além da pesquisa; as políticas de idiomas (BERNINI, 2015; HAMEL; LÓPEZ; CARVALHAL, 2016) a internacionalização curricular (LEASK; BRIDGE, 2013; LEAL; MORAES, 2016; STALLIVIERI, 2016; PRIETO-FLORES; FEU; CASADEMOND, 2016), entre outros aspectos. Complementarmente, o próprio conceito de cooperação internacional implícito nas avaliações é reducionista, uma vez que elas igualam cooperação a publicações conjuntas (MARGINSON, 2017).

Infere-se, portanto, que a forma como a internacionalização tem sido medida pelos rankings acadêmicos apresenta limitações significativas, resultantes do reducionismo e da simplificação de algo complexo, dinâmico e multifacetado. Tais ferramentas parecem se limitar ao cotejamento de um percentual reduzido de dimensões decorrentes dos processos institucionais de internacionalização, sendo avaliados apenas quantitativamente e reforçando o instrumentalismo econômico que permeia a internacionalização da educação superior global. Cabe lembrar que, como ação institucional abrangente, a internacionalização implica em

\footnotetext{
um compromisso, confirmado através da ação, para infundir perspectivas internacionais e comparativas no ensino, na pesquisa e nos demais serviços da educação superior. Ela molda o ethos e os valores institucionais e afeta toda a instituição. É essencial que ela seja abraçada pelas lideranças institucionais, gestores, professores, estudantes e todas as unidades de serviço e suporte acadêmico (HUDZIK, 2011, p. 6, tradução nossa).
}

De Wit $(2015,2016)$ complementa que a internacionalização deve estar alinhada à missão universitária, sendo, portanto, compreendida como um meio e não como um fim em si mesma. Entende-se, portanto, que à medida que as universidades reduzirem suas políticas e estratégias de internacionalização a critérios exclusivamente quantitativos, voltadas à manutenção ou à elevação de seu posicionamento nos rankings, elas deixam de lado seu compromisso maior com a qualidade da educação e com sua entrega à sociedade.

\section{CONSIDERAÇÕES FINAIS}

Este artigo se propôs a analisar os principais rankings acadêmicos da atualidade, com foco nos indicadores relacionados à internacionalização da educação superior. Foram cotejados seis rankings acadêmicos, sendo três internacionais e três nacionais. Evidenciou-se que cinco

n.1

p.52-73


dessas ferramentas contemplam indicadores explicitamente relacionados à internacionalização da educação superior e que um deles o faz de maneira indireta. A pesquisa internacional constituiu-se como o indicador de internacionalização mais significativo para os rankings abrangidos no recorte deste estudo.

Optou-se por não se ater às questões epistemológicas inerentes ao debate sobre os rankings acadêmicos - como, por exemplo, se as universidades devem ou não ser ranqueadas e a quais interesses essas ferramentas servem. No entanto, os resultados aqui evidenciados corroboram com o entendimento de que existem limitações significativas, tanto em termos filosóficos quanto pragmáticos, em relação ao uso dos rankings acadêmicos como único parâmetro para a tomada de decisão de estudantes, docentes e pesquisadores, bem como para a formulação de políticas de internacionalização e de gestão.

Cada um desses processos de ranqueamento apresenta fraquezas técnicas e metodológicas próprias, sendo que, até o momento, nenhum deles conseguiu contemplar a totalidade das missões e dos objetivos que permeiam instituições universitárias ao redor do mundo. A extensão, por exemplo, considerada uma das finalidades da educação superior nos países latino-americanos, tem sido totalmente negligenciada, prejudicando contextos com projetos significativos nesse sentido. Ademais, ainda que a cada nova publicação os rankings acadêmicos incorporem novos critérios de avaliação, com vistas ao aperfeiçoamento metodológico, eles ainda seguem um padrão predominantemente quantitativo, inclusive no que se refere aos indicadores de internacionalização. Essa incorporação de novos critérios, inclusive, os leva a não recomendarem a comparação de resultados com suas publicações anteriores (THE, 2016), o que inviabiliza análises longitudinais a respeito de seus resultados.

Complementarmente, os indicadores e os pesos adotados não estão orientados segundo justificações teóricas. Desse modo, eles implicam em certa superficialidade, além de produzirem um "efeito de persistência" com caráter elitista em relação às instituições mais bem classificadas, "privilegiando os que já são privilegiados" (ALTBACH, 2015, p. 3, tradução nossa) ou "reciclando a dominação das universidades mais fortes" (MARGINSON, 2017, p. 7, tradução nossa), visto que, na ausência de uma análise qualitativa e contextualizada, universidades menores dificilmente alcançarão o topo das listas, mesmo que estejam bem adequadas às suas finalidades institucionais. Como a Unesco (2013) alerta, apenas $1 \%$ das universidades do mundo são incluídas nos rankings.

Por fim, são contraditórios os fatos de que os global rankings mais utilizados na atualidade como o Q\&S e o THE - não tenham quaisquer vínculos diretos com o meio acadêmico e de que, em alguns casos, os próprios rankings ofereçam consultorias às instituições universitárias para que elas possam alcançar melhores posicionamentos. 
Uma vez que os rankings acadêmicos parecem inevitáveis na atual conjuntura (O'CONNELL; SAUNDERS, 2012; LIU, 2015; ALTBACH, 2015; MARGINSON, 2017), é relevante que um olhar dos gestores universitários e dos policymakers envolvidos no contex to da educação superior esteja direcionado aos possíveis desdobramentos da elevada ênfase conferida a essas ferramentas, inclusive no que se refere aos planos institucionais de internacionalização. Como alguns relatórios de organismos internacionais e pesquisadores já evidenciaram, os rankings tendem a levar as universidades e os centros de pesquisa - que, ao menos em princípio, não têm o lucro como principal função - a adquirem um comportamento predominantemente empresarial, com foco em eficiência, produtividade e competitividade. Cabe lembrar, portanto, que, em última instância, os rankings acadêmicos são uma espécie de avaliação de reputação e prestígio, que não necessariamente refletem a qualidade das instituições avaliadas.

\section{REFERÊNCIAS}

AGUILLO, Isidro. Rankings de universidades: el ranking Web. Higher Learning Research Communication, v. 2, n. 1, p. 3-12. 2012. Disponível em: < http://files.eric.ed.gov/fulltext/ EJ1134275.pdf >. Acesso em: 6 out. 2017.

ALTBACH, Phillip. The dilemmas of ranking. International Higher Education, n. 42, p. 23, 2015. Disponível em: < https://ejournals.bc.edu/ojs/index.php/ihe/article/view/7878/7029 >. Acesso em: 6 out. 2017.

ALTBACH, Phillip; HAZELKORN, Ellen. Pursuing rankings in the age of massification: for most- forget about it. International Higher Education, n. 89, p. 8-10, Spring 2017.

Disponível em: < https://ejournals.bc.edu/ojs/index.php/ihe/article/view/9834/8616>. Acesso em: 6 out. 2017.

ACADEMIC RANKING OF WORLD UNIVERSITIES (ARWU). ARWU 2015:

metodologia. 2016. Disponível em: < http://www.shanghairanking.com/pt/aboutarwu.html >. Acesso em: 8 set. 2016.

BERNINI, Giuliano. For a language policy in the internationalized university. Journal of Technology Transfer, v. 40, n. 3, p. 380-386. 2015. Disponível em: < https://link.springer.com/article/10.1007/s10961-014-9351-1>. Acesso em: 6 out. 2017.

BUELA-CASAL, Gualberto; GUTIÉRREZ-MARTÍNEZ, Olga; BERMÚDEZ-SÁNCHEZ, María Paz; VADILLO-MUÑOZ, Oscar. Comparative study of international academic rankings of universities. Scientometrics, v. 71, n. 3, p. 349-365, 2007. Disponível em: < https://link.springer.com/article/10.1007\%2Fs11192-007-1653-8>. Acesso em: 6 out. 2017. 
DE WIT, Hans. Is the international university the future for higher education? International Higher Education, n. 80, Spring 2015. Disponível em: < https://ejournals.bc.edu/ojs/index.php/ihe/article/view/6133/5372>. Acesso em: 6 out. 2017.

DE WIT, Hans. The impact of rankings on internationalization. IREG-8 Conference, Lisbon, Portugal. 4-6 May 2016.

GUIA DO ESTUDANTE. Conheça a trajetória do Guia do Estudante. 2016. Disponível em: 〈http://guiadoestudante.abril.com.br/premio/sobre-premio/conteudo_132818.shtml >. Acesso em: 1 out 2016.

GUIA DO ESTUDANTE. Prêmio Melhores Universidades Guia do Estudante 2015. 2016a. Disponível em: < http://guiadoestudante.abril.com.br/vestibular-enem/escolas-notadez-premio-melhores-universidades-guia-estudante-2015-905103.shtml >. Acesso em: 1 out. 2016.

GUIA DO ESTUDANTE. Entenda como é feita a avaliação de cursos do Guia do Estudante. 2016b. Disponível em: <http://guiadoestudante.abril.com.br/vestibularenem/entenda-como-feita-avaliacao-guia-estudante-950288.shtml >. Acesso em: 1 out. 2016.

HAMEL, Rainer Enrique; LÓPEZ, Elisa Álvarez; CARVALHAL, Tatiana Pereira. Language policy and planning: challenges for Latin American universities. Language Planning, v. 17, n. 3-4, p. 278-297. 2016. Disponível em: < http://www.tandfonline.com/doi/abs/

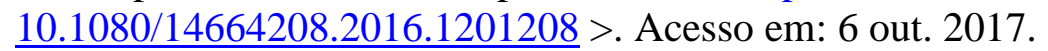

HUDZIK, J. Comprehensive internationalization. Washington, DC: NAFSA, 2011.

IREG: OBSERVATORY ON ACADEMIC RANKING AND EXCELLENCE. IREG-8 Conference. University rankings and International Academic Relations: a bridging tool oh hindrance? 2016. Disponível em: < http://ireg-observatory.org/en/ireg-8-programme >. Acesso em: 16 mar 2017.

JONS, Heike; HOYLER, Michael. Global geographies of higher education: the perspective of world university rankings. Geoforum, v. 46, p. 45-59, 2013. Disponível em: < http://www.sciencedirect.com/science/article/pii/S0016718512002886>. Acesso em: 6 out. 2017.

KIM, Terri. Academic mobility, transnational identity capital, and stratification under conditions of academic capitalism. Higher Education, v. 73, n. 6, p. 981-997, 2017. Disponível em: < https://link.springer.com/article/10.1007/s10734-017-0118-0>. Acesso em: 6 out 2017.

KNIGHT, Jane. Internationalization remodeled: definition, approaches, and rationales. Journal of Studies in International Education, v. 8, n. 1, 5-31, 2004. Disponível em: < http://journals.sagepub.com/doi/pdf/10.1177/1028315303260832>. Acesso em: 6 out 2017. 
KNIGHT, Jane. Student mobility and internationalization: Trends and tribulations. Research in Comparative and International Education, v. 7, n. 1, p. 20-33, 2012. Disponível em: < http://journals.sagepub.com/doi/pdf/10.2304/rcie.2012.7.1.20>. Acesso em: 6 out. 2017.

KNIGHT, Jane. International universities: misunderstandings and emerging models? Journal of Studies in International Education, v. 19, n. 2, p. 1-15. 2015. Disponível em: < http://journals.sagepub.com/doi/abs/10.1177/1028315315572899>. Acesso em: 6 out. 2017. LAUS, Sônia. A internacionalização da educação superior: um estudo de caso da Universidade Federal de Santa Catarina. 2012. Tese (Doutorado em Administração). Universidade Federal da Bahia, Salvador, 2012.

LEAL, Fernanda Geremias; MORAES, Mário César Barreto. Internacionalização do currículo: um olhar crítico fundamentado no pensamento complexo. In: LUNA, Marcelo. (Org.). Internacionalização do currículo: educação, interculturalidade, cidadania global. São Paulo: Pontes, 2016. Disponível em: < https://www.researchgate.net/publication/ 303186963_Internacionalizacao_do_curriculo_Um_olhar_critico_fundamentado_no_pensam ento_complexo>. Acesso em: 6 out 2017.

LEASK, Betty; BRIDGE, Christopher. Comparing internationalisation of the curriculum in action across disciplines: theoretical and practical perspectives. Compare: a journal of comparative and international education, v, 43, n. 1, p. 79-101. 2013. Disponível em: < https://federation.edu.au/_data/assets/pdf_file/0015/200409/Comparing-IoC_Leask-andBridge2013.pdf >. Acesso em: 6 out 2017.

LIMA, Manolita Correia; CONTEL, Fábio Betiolli. Internacionalização da educação superior: nações ativas, nações passivas e a geopolítica do conhecimento. São Paulo: Alameda, 2011.

LIU, Nian. Cai. The story of Academic Ranking of World Universities. International Higher Education, Chestnut Hill, MA, v.54, p. 2-3, Winter 2015. Disponível em: < https://ejournals.bc.edu/ojs/index.php/ihe/article/view/8409/7543>. Acesso em: 6 out 2017.

MARGINSON, Simon. Global university ranking and performance improvement: what kind of international academic relations are created by rankings? IREG - CONFERENCE, 8.: 2016, Lisboa. [Proceedings...]. Lisboa: Universidade Nova de Lisboa, 2016.

MARGINSON, Simon. Do rankings drive better performance? International Higher Education, n. 89, Spring 2017. Disponível em: < https://ejournals.bc.edu/ojs/ index.php/ihe/article/view/9833/8615 >. Acesso em: 6 out 2017.

MARINGE, Felix; FOSKETT, Nick; WOODFIELD, Steve. Emerging internationalisation models in an uneven global terrain: findings from a global survey. Compare: a Journal of Comparative and International Education, v. 43, n. 1, p. 9-36, 2013.

MORAES, Mário César Barreto. 503 dúvidas para você tirar sobre a educação superior no Brasil. Florianópolis: Naus, 2016.

\begin{tabular}{|l|c|c|c|c|c|}
\hline C Rev. Inter. Educ. Sup. & Campinas, SP & v.4 & n.1 & p.52-73 & jan./abr. 2018 \\
\hline
\end{tabular}


NUNES, Edson; FERNANDES, Ivanildo. Rankings internacionais: a irresistível polêmica em torno de seus sentidos e metodologias. Revista Ensino Superior, Campinas, n.12, jan./mar. 2014. Disponível em: < https://www.revistaensinosuperior.gr.unicamp.br/artigos/rankingsinternacionais-a-irresistivel-polemica-em-torno-de-seus-sentidos-e-metodologias $>$. Acesso em: 6 out 2017.

O'CONNELL, Catherine; SAUNDERS, Murray. Mediating the use of global university rankings: perspectives from education facilitators in an international context. Journal of Studies in International Education, v. 17, n. 4, p. 354-376. 2012. Disponível em: < http://journals.sagepub.com/doi/pdf/10.1177/1028315312453743 >. Acesso em: 6 out 2017.

PÉREZ-ESPARRELLS, Carmen; LOPEZ GARCÍA, Ana. Rankings de instituciones de educación superior: panorama internacional. Calidad em la Educación, v. 30, p. 328-343. 2009.

PÉREZ-ESPARRELLS, Carmen; GÓMEZ-SANCHO, Jose Maria. Los rankings internacionales de las instituciones de educación superior y las clasificaciones universitarias en España: visión panorámica y prospectiva de futuro. Fundación de Las Cajas de Ahorros, 2010. Disponível em: 〈http://www.univnova.org/documentos/440.pdf >. Acesso em: 6 out. 2017.

PRIETO-FLORES, Òscar; FEU, Jordi; CASADEMONT, Casademond. Assessing intercultural competence as a resulto $f$ internationalization at home efforts: a case study from the Nightingale Mentoring Program. Journal of Studies in International Education, n. 20, v. 50, p. 437-453. 2016. Disponível em: < http://journals.sagepub.com/doi/pdf/ 10.1177/1028315316662977 >. Acesos em: 6 out. 2017.

QUACQUARELLI SYMONDS (Q\&S). Q\&S University rankings. Disponível em: < http://www.iu.qs.com/university-rankings>. Acesso em: 9 set. 2016. 2016.

RANKING UNIVERSITÁRIO FOLHA (RUF). O que é o RUF. 2016. Disponível em: < http://ruf.folha.uol.com.br/2015/o-ruf/>. Acesso em: 9 set. 2016.

RANKING UNIVERSITÁRIO FOLHA (RUF). Como é feito o ranking de universidades. 2016a. Disponível em: < http://ruf.folha.uol.com.br/2015/o-ruf/ranking-universidades/>. Acesso em: 9 set. 2016.

STALLIVIERI, Luciane. Estratégias para a internacionalização do currículo: do discurso à prática. In: Luna, Marcelo (Org.). Internacionalização do currículo: educação, interculturalidade, cidadania global. São Paulo: Pontes, 2016.

STALLIVIERI, Luciane. Internacionalização e intercâmbio: dimensões e perspectivas. Curitiba: Appris, 2017. 
TIMES HIGHER EDUCATION (THE). About. 2016. Disponível em: < https://www.timeshighereducation.com/world-university-rankings/about-the-times-highereducation-world-university-rankings>. Acesso em: 9 set. 2016.

TIMES HIGHER EDUCATION (THE). Methodology. 2016a. Disponível em: < https://www.timeshighereducation.com/news/ranking-methodology-2016>. Acesso em: 9 set. 2016.

TIMES HIGHER EDUCATION (THE). Brand u: how colleges and universities nurture their reputations. 2016b. Disponível em: < https://www.timeshighereducation.com/worlduniversity-rankings/times-higher-education-to-host-symposium-on-university-reputation-andbrands >. Acesso em: 16 abr. 2017.

UNESCO. Rankings and accountability in higher education: uses and misuses. UNESCO, 2013.

VIEIRA, Rosilene; LIMA, Manolita Correia. Academic rankings: from its genesis to its international expansion. Higher Education Studies, v. 5, n. 1, p. 63-72. 2015. Disponível em: < http://www.ccsenet.org/journal/index.php/hes/article/view/42347/24161>. Acesso em: 6 out. 2017.

VIEIRA, Rosilene; LIMA, Manolita Correia. A busca pelo selo "universidade classe do mundo" e os rankings acadêmicos globais pioneiros. Interfaces Brasil/Canadá, Canoas, v. 16, n. 1, 97-116. 2016. Disponível em: < https://periodicos.ufpel.edu.br/ojs2/index.php/ interfaces/article/view/7704/5616>. Acesso em: 6 out. 2017.

\footnotetext{
${ }^{\mathrm{i}}$ Sobre os autores

Fernanda Geremias Leal

E-mail: fernanda.leal@ufsc.br / ORCID: http://orcid.org/0000-0002-1716-2060

Universidade do Estado de Santa Catarina (UDESC) - Brasil

Doutoranda em Administração - UDESC.

Luciane Stallivieri

E-mail: lustalliv@gmail.com / ORCID: http://orcid.org/0000-0002-2104-8607

Universidade Federal de Santa Catarina (UFSC) - Brasil

Pós-doutoranda em Engenharia de Produção - UFSC

Mário César Barreto Moraes

E-mail: mcbmstrategos@gmail.com / ORCID: http://orcid.org/0000-0002-0760-8444

Universidade do Estado de Santa Catarina (UDESC) - Brasil

Doutor em Engenharia de Produção - UFSC
} 